\title{
Metal-Depleted Brown Dwarfs
}

Nicolas Lodieu

\begin{abstract}
This chapter reviews our current knowledge of metal-poor ultracool dwarfs with spectral types later than M7. The current census of M, L, and T subdwarfs is explored. The main colour trends of subdwarfs from the optical to the mid-infrared are described and their spectral features presented, which led to a preliminary and tentative spectral classification subject to important changes in the future when more of these metal-poor objects are discovered. Their multiplicity and the determination of their physical parameters (effective temperature, gravity, metallicity, and mass) are discussed. Finally, some suggestions and future guidelines are proposed to foster our knowledge on the oldest and coolest members of our Galaxy.
\end{abstract}

\section{Introduction}

M dwarfs represent the majority of stars in the solar neighbourhood (Kirkpatrick et al.|2012) and in our Galaxy where the mass function peaks (e.g. Chabrier 2003). At lower masses, three new classes have defined during the past two decades: the L dwarfs whose atmospheres are affected by dust (Kirkpatrick et al. 1999; Martín et al. 1999), the T dwarfs shaped by methane and water absorption bands (Leggett et al. 2000, Geballe et al. 2002; Burgasser et al. 2002, 2006), and the Y dwarfs with the potential presence of ammonia at infrared wavelengths (Cushing et al. 2011, Kirkpatrick et al. 2012). The classification of $\mathrm{L}$ dwarfs is mostly morphological but the large variety of sources discovered in optical and infrared large-scale surveys triggered a preliminary spectral scheme incorporating a new parameter: gravity (i.e. ages) as proposed by two independent teams (Cruz et al. 2009, Allers et al. 2007, Allers and Liu 2013). However, the spectral classification of metal-poor L dwarfs remains in its infancy due to the small sample existing in the literature. Nonetheless, recent discoveries offered new hints to elaborate a tentative spectral sequence.

Instituto de Astrofísica de Canarias, C. Via Lactea S/N, E-38205 La Laguna, Tenerife, Spain, email: nlodieudiac.es 
Metal-poor dwarfs belong to the spectral class VI in the Morgan-Keenan scheme (Morgan et al. 1943). They are also known as subdwarfs and often abbreviated "sd" (Joy 1947; Gizis 1997; Lépine et al.|2007). They usually exhibit bluer optical and infrared colours than their solar-like analogues (Lodieu et al. 2017) and show distinct spectral features such as the weakening of $\mathrm{TiO}$ bands (i.e. less $\mathrm{TiO}$ opacity implying more flux radiated from deeper and hotter layers of the atmosphere), strengthening of $\mathrm{CaH}$ bands, and strong collision-induced hydrogen absorption beyond $1 \mu \mathrm{m}$ (Gizis 1997; Lépine et al. 2007). They typically have high proper motions and large radial velocities translating into space motions compatible with membership to the thick disk and halo (Schmidt 1975). This population of metal-poor dwarfs is important for several reasons. Firstly, they represent key tracers of the history of our Galaxy because they are very old. Secondly, the knowledge of their physical parameters will impact on the study of globular clusters whose main populations are metal-poor and old. Thirdly, the census of metal-poor stars and brown dwarfs helps the determination of the luminosty and mass functions early on in the formation of our Galaxy to gauge the impact of metallicity in star formation processes. Unfortunately, metal-poor stars are not so numerous compared to their solar-like counterparts with only three subdwarfs of the $\sim 250$ systems located within $10 \mathrm{pc}$ ( $\mu$ Cas AB; Kapteyn's star; CF Uma).

This review will focus mainly on ultracool subdwarfs (UCSDs) with spectral types later than $\mathrm{M} 7$ and metallicities $(\mathrm{Fe} / \mathrm{H})$ equal or less than -0.5 dex unless otherwise stated. For more massive subdwarfs, readers are referred to one of the section dedicated to M subdwarfs in the book of Reid and Hawley (2005). The coolest Ltype subdwarfs might be brown dwarfs but none of them has been unambiguously proven to be substellar at the time of writing (Lodieu et al. 2015). A few T-type metal-poor dwarfs have been announced as companions to bright stars with welldetermined metallicities (Pinfield et al. 2012; Burningham et al. 2013) but only one has a metallicity below -0.5 dex, WISE J20052038+5424339 (Mace et al.|2013).

This review summarizes the techniques employed over the past decades to identify metal-poor low-mass stars and brown dwarfs, and describes the main spectral features leading to a preliminary and tentative classification scheme for UCSDs. The colours of UCSDs are mentioned and compared to those of nearby field M and L dwarfs. Our current knowledge on the multiplicity of UCSDs and the actual estimates of the physical parameters of the lowest mass metal-poor stars and brown dwarfs are also presented here. Finally, future needs are highlighted to characterise in more details the population of UCSDs and their physical parameters.

\section{Census of ultracool subdwarfs}

Dedicated searches for metal-poor stars and brown dwarfs usually focus on proper motion surveys to bias their final sample towards high velocity objects, thus halo stars (Schmidt 1975). Most of the late-M subdwarfs have been identified in photographic plates from the Digital Sky Survey and the SuperCOSMOS Sky Survey 
(Gizis 1997, Gizis and Reid 1997; Gizis et al.| 1997; Schweitzer et al. 1999; Lépine et al. 2003; Scholz et al. 2004a b, Lodieu et al. 2005; Lépine et al. 2007), and more recent all-sky or large-scale surveys such as the Two Micron All Sky Survey (2MASS; Burgasser and Kirkpatrick 2006; Cushing et al. 2009), the Sloan Digital Sky Survey (SDSS; Lépine and Scholz 2008; Sivarani et al. 2009; Zhang et al. 2013), the UKIRT Infrared Deep Sky Survey (UKIDSS; Lodieu et al.|2012, 2017), and the Wide field Infrared Survey Explorer (WISE; Kirkpatrick et al. 2014, 2016).

Over the past years, the number of L subdwarfs has grown rapidly and is now just slightly over 30. The first one was identified in 2MASS (Burgasser et al.|2003) followed by other discoveries in the same database (Burgasser 2004: Cushing et al. 2009), SDSS (Sivarani et al. 2009; Schmidt et al.|2010; Bowler et al.|2010), WISE (Kirkpatrick et al. 2014, 2016), and cross-correlations of various surveys (Lodieu et al. 2010, 2012, 2017, Zhang et al.|2017b a).

In the $\mathrm{T}$ dwarf regime, a few examples of metal-poor $\mathrm{T}$ dwarfs have been reported as companions to brighter stars with well determined metallicities both in UKIDSS (Pinfield et al. 2012; Burningham et al. 2013) and WISE (Mace et al. 2013). Nonetheless, their metallicities generally do not exceed -0.5 dex, except in the case of WISE J200520+542433 with $\mathrm{Fe} / \mathrm{H}=-0.64$ dex (Mace et al. 2013).

\section{Colours}

Due to the dearth of metals in their atmospheres, UCSDs tend to exhibit on average bluer colours than their solar-type analogues (Fig. 1). Moreover, they lie below solar-metallicity dwarfs in reduced proper motions (left panel in Fig. 1) which constitute powerful tools to identify UCSDs (Lépine et al. 2007; Lépine and Scholz 2008; Kirkpatrick et al. 2016, Lodieu et al. 2017). This section summarises differences reported in the literature going from blue to red wavelengths. These differences helped out increasing the numbers of UCSDs over the past years and represent key indices for upcoming surveys like the Large Synoptic Survey Telescope (LSST; Ivezic et al.2008) and the Euclid mission (Mellier 2016).

- Their locus in a $(g-r, r-i)$ colour-colour diagram is distinct from their solartype counterparts. Selecting sources with $g-r>2$ mag and $g-i>3$ mag will strongly bias the photometric selection towards metal-poor M dwarfs (Fig. 2 in Lépine and Scholz|2008).

- Their $r-z$ colours are bluer than field $\mathrm{M}$ dwarfs by at least 1 mag (Fig. 2 in Lépine and Scholz 2008).

- The optical-to-infrared colours (e.g. $i-J)$ of UCSDs are bluer as metallicity decreases, yielding a flattening of the far-red part of their optical spectra (Gizis 1997; Lépine et al. 2007; Lodieu et al. 2017; Zhang et al. 2017b).

- Their infrared colours (e.g. $J-K<0.7 \mathrm{mag}$ ) are much bluer than solar-type M dwarfs due to the strong pressure-induced $\mathrm{H}_{2}$ opacity beyond 1 micron (Lodieu et al. 2017, Zhang et al.2017b). 
- Subdwarfs of type M and L typically fall bluewards in near-infrared to midinfrared colours (e.g. $J-w 1, J-w 2, H-w 2$ ) of their solar-metallicity counterparts due to the increasing influence of collision-induced hydrogen absorption (Kirkpatrick et al. 2016; Lodieu et al. 2017).

- Their mid-infrared colours look similar to those of their solar-type analogues although opposite trends have been reported in the literature: red in Kirkpatrick et al. (2016) and blue in Lodieu et al. (2017).
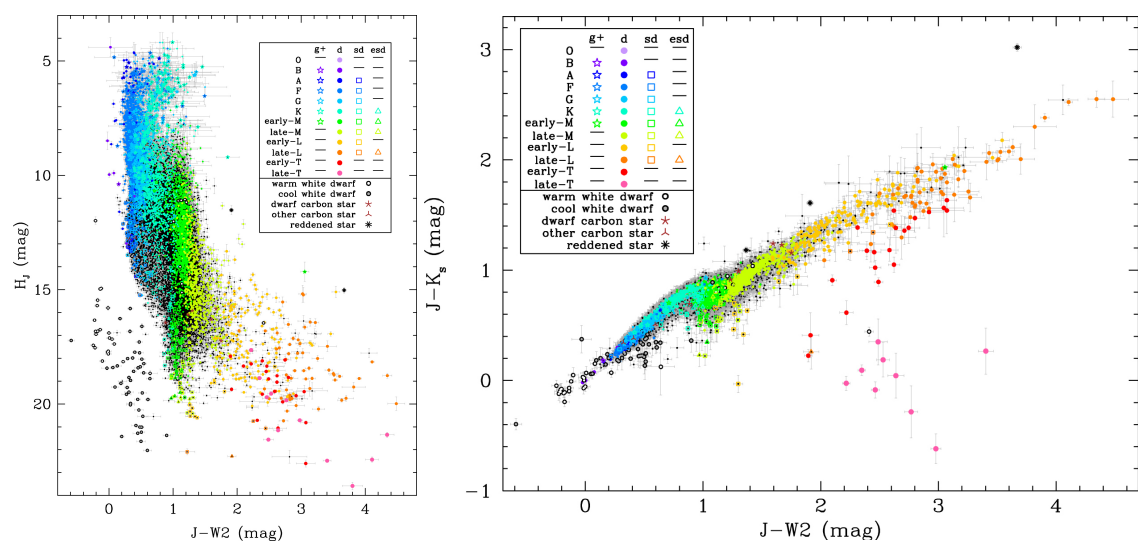

Fig. 1 Reduced proper motion (left) and $\left(J-w 2, J-K_{s}\right)$ colour-colour diagrams for main-sequence stars, M, L, and T dwarfs of different metallicities. Figures taken Kirkpatrick et al. (2016).

\section{Spectral features}

The main spectral features indicative of low metallicity are the strengthening of the $\mathrm{CaH}$ bands and weakening of the TiO bands around 620-740 nm and the effect of the collision-induced absorption beyond $1000 \mathrm{~nm}$. However, there are other features which can be employed to distinguish UCSDs from their solar-type cousins over the optical-infrared wavelength range (Gizis 1997; Lépine et al. 2007; Burgasser et al. 2007: Kirkpatrick et al. 2016; Zhang et al. 2017b), as enumerated below:

1. The $\mathrm{CaH}$ bands around $640-700 \mathrm{~nm}$ is stronger with lower metallicity but is also dependent on temperature in the 3600-3200 K range.

2. The TiO bands at $\sim 720 \mathrm{~nm}, 780 \mathrm{~nm}$, and $850 \mathrm{~nm}$ are weaker with lower metallicity. The bluest of these bands, however, becomes more sensitive to temperature than metallicity for late-type M subdwarfs. Below $3200 \mathrm{~K}$, the strength of the $\mathrm{TiO}$ band at $720 \mathrm{~nm}$ is not monotonic anymore with decreasing metallicity, making the classification of late-M and early-L subdwarfs based on spectral indices more unreliable. 
3. The VO band at $800 \mathrm{~nm}$ is a strong indicator of metallicity in L dwarfs: it weakens as $\mathrm{Fe} / \mathrm{H}$ decreases.

4. The $\mathrm{CO}$ band at $2300 \mathrm{~nm}$ weakens as metallicity decreases and eventually disappears in extreme and ultra-subdwarfs.

5. The near-infrared flux beyond $1000 \mathrm{~nm}$ becomes more depressed with lower metallicity due to the strong collision-induced $\mathrm{H}_{2}$ absorption.

\section{Spectral types}

The first spectral classification of M subdwarfs has been proposed by Gizis (1997), dividing $M$ dwarfs into three main classes: solar-type $M$ dwarfs with metallicity $\mathrm{Fe} / \mathrm{H}$ around $0, \mathrm{M}$ subdwarfs (sdM) with $\mathrm{FeH} \sim-1.2 \pm 0.3$ dex, and extreme $\mathrm{M}$ subdwarfs (esdM) with approximate $\mathrm{Fe} / \mathrm{H}$ of $-2.0 \pm 0.5 \mathrm{dex}$. This classification scheme is based on the strength of the $\mathrm{TiO}$ and $\mathrm{CaH}$ bands in the 620-740 nm optical range. Spectral indices have been defined to infer both metal class and spectral type. Ten years later, Lépine et al. (2007) revised the boundaries of the original metal classes based on a larger sample (factor of five bigger) of known metal-poor single and multiple systems. They introduced a new parameter, $\tau_{T i O / C a H}$, as well as an additional metal class with metallicities even lower than the esdM, the ultrasubdwarfs (usdM), to taken into account the positions of known binaries sharing the same metallicity in the (early-)M dwarf regime. They also proposed spectral standards for each metal class and spectral types ranging from M0 to M8. The quality of the optical spectra of the sdM, esdM, and usdM templates has been improved by Savcheva et al.(2014) by stacking all SDSS spectra available per subtype and per metal class (Fig. 2). Jao et al. (2008) presented new thoughts about the naming and spectral classification of subdwarfs based on a sample of $88 \mathrm{~K} 3-\mathrm{M} 6$ sources. They propose to use the class VI of the Morgan-Keenan scheme instead of the terminology "sd" to name subdwarfs, a prefix that can be confused with the hotter sdO/sdB-type star which also appear sub-luminous in the HR diagram. And they showed the influence of gravity in the spectra of M subdwarfs and suggested to avoid classification based solely on spectral indices.

In the $\mathrm{L}$ dwarf, the current classification is only tentative due to the small number of $\mathrm{L}$ subdwarfs announced to date and the narrow range of physical parameters. Nonetheless, several groups attempted to extend the $\mathrm{M}$ dwarf classification into the $\mathrm{L}$ regime. Zhang et al. (2017b) built on the extensions proposed by Burgasser et al. (2007) and Kirkpatrick et al. (2016), keeping the concept of the three metal classes proposed by Lépine et al. (2007) and applying it to the L subdwarfs (sdL, esdL, and usdL). Their spectral classification is based on the comparison of spectra of about 30 L0-L8 subdwarfs with those of solar-type L dwarf standards defined in the literature (e.g. Kirkpatrick et al. 2000). They focused on key features sensitive to metallicity and temperatures (their Table 3 and previous section) to evaluate the differences in the optical (CaH and VO bands around $700 \mathrm{~nm}$, VO band at $800 \mathrm{~nm}$, strength of the KI doublet at $770 \mathrm{~nm}, \mathrm{TiO}$ band at $850 \mathrm{~nm}$ ) and in the infrared $(\mathrm{FeH}$ band at 
$990 \mathrm{~nm}, \mathrm{CO}$ band at $2300 \mathrm{~nm}$, and the depression in the $H$ and $K$ passbands due to the collision-induced $\mathrm{H}_{2}$ absorption), yielding a revised classification of known metal-poor $\mathrm{L}$ dwarfs that can be used for future discoveries (Fig. 3).
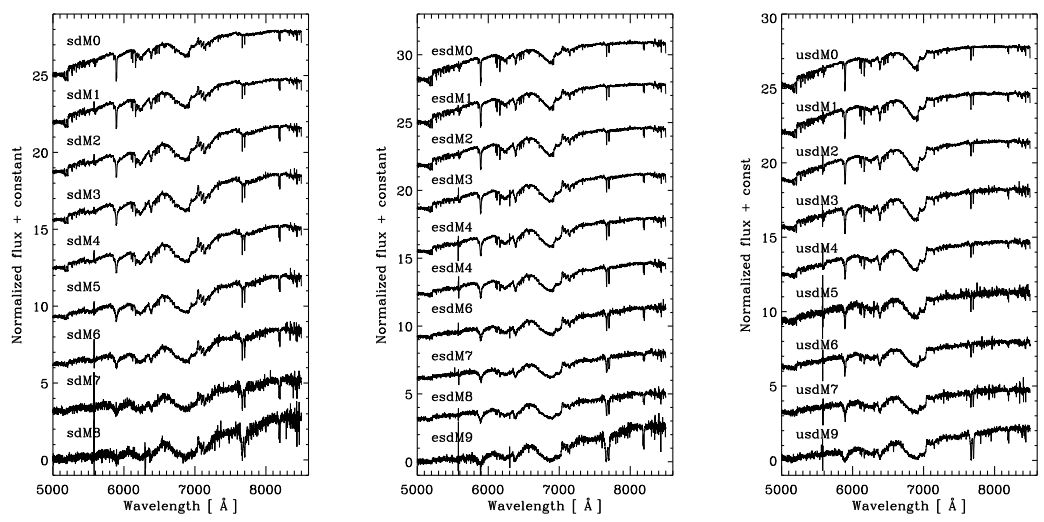

Fig. 2 Sequence of M-type subdwarfs (sdM; left), extreme subdwarfs (esdM, middle), and ultrasubdwarfs (usdM; right). Figure taken from Savcheva et al. (2014) with an earlier version shown in Lépine et al. (2007).

\section{Multiplicity}

The multiplicity fraction and binary properties of UCSDs is poorly constrained for two main reasons. On the one hand, most of the known UCSDs have been identified very recently and, on the other hand, they are faint both at optical and infrared wavelengths. High-resolution imaging is feasible for a limited sub-sample because bright reference stars are needed to close the loop as in the case of adaptive optics for example.

Only a limited number of surveys have been conducted to look at the multiplicity of metal-poor M dwarfs over a wide range of separations. Jao et al. (2009) found that the multiplicity rate of $\mathrm{K}$ and $\mathrm{M}$ subdwarfs is slightly lower than their solar-type counterparts $(26 \pm 6 \%$ vs $36 \pm 5 \%$ ) from an optical speckle survey. The total multiplicity fraction of $\mathrm{M}$ subdwarfs can be divided up as follows: $3 \%$ have companions within $10 \mathrm{au}$, another $3 \%$ within the $10-100$ au range, $14 \%$ beyond $100 \mathrm{au}$, and the remaining $6 \%$ are spectroscopic binaries. Combining the outcome of a Hubble survey of 28 metal-poor M dwarfs of Riaz et al.(2008) with high spatial lucky imaging observations of $24 \mathrm{M}$ subdwarfs, Lodieu et al. (2009) resolved only one system with a projected separation of 0.7 arcsec (LHS 182), deriving a binary frequency of $3.7 \pm 2.6$ ( $1 \sigma$ confidence limit) for M subdwarfs (mainly M0-M5). This result is in 


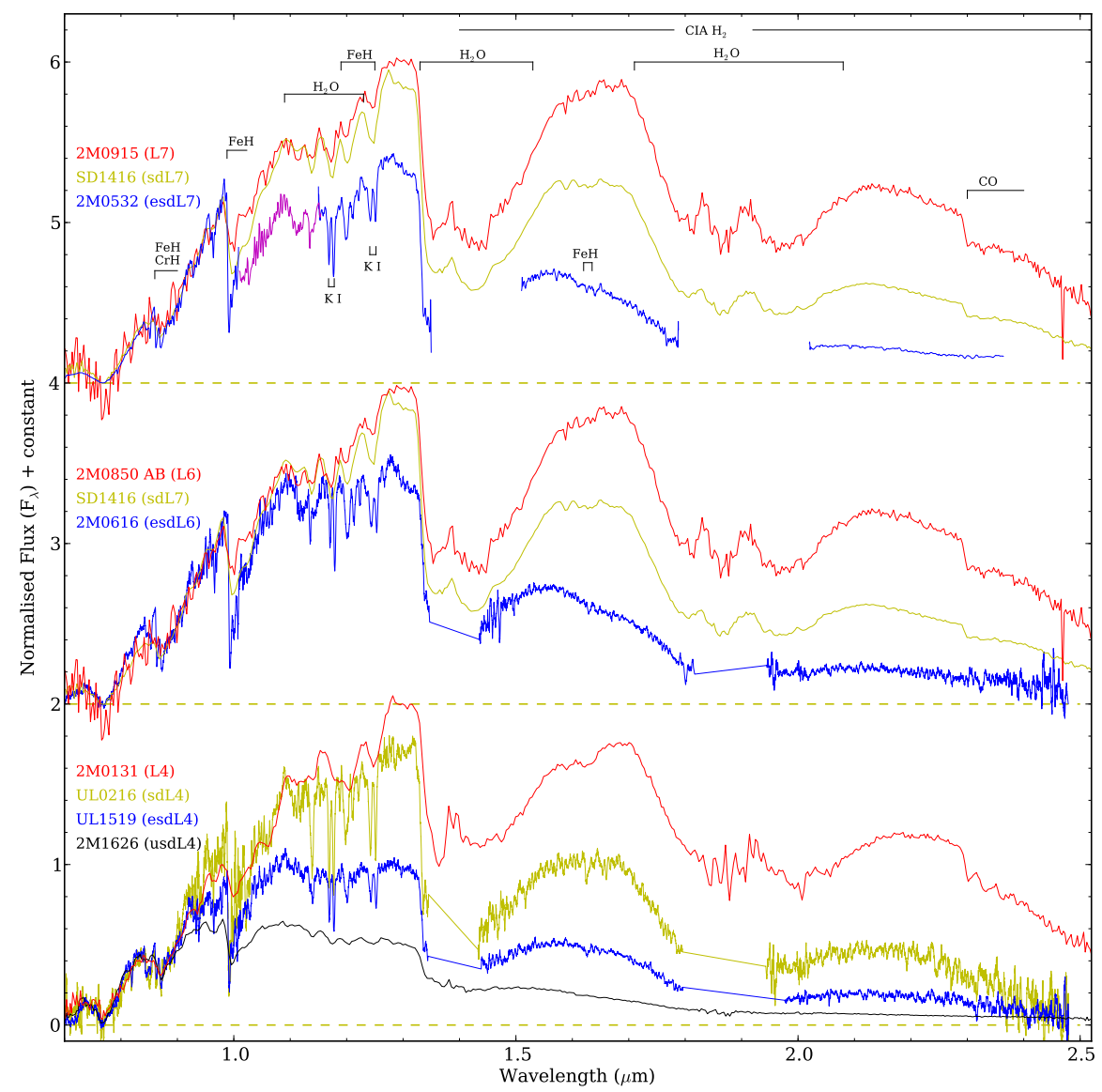

Fig. 3 Optical and near-infrared spectra of L4, L6, and L7 dwarfs/subdwarfs with different subclasses. Spectra have been normalised at $0.89 \mu \mathrm{m}$. The missing wavelength region in the spectrum of $2 \mathrm{M} 0532(1.008-1.153 \mu \mathrm{m})$ has been replaced by the best BT-Settl model fit in magenta $\left(\mathrm{T}_{\text {eff }}=1600 \mathrm{~K},[\mathrm{Fe} / \mathrm{H}]=1.6 \mathrm{dex}\right.$, and $\left.\log (\mathrm{g})=5.25 \mathrm{dex}\right)$. Figure from Zhang et al. (2017b).

line with the two companions exhibiting $\mathrm{H} \alpha$ in emission mong a sample of 68 LHS objects (2.9\%; Gizis 1998).

Finally, only three $\mathrm{M}$ subdwarfs with metallicities below -0.5 dex and masses less than $0.5 \mathrm{M}_{\odot}$ have dynamical mass measurements. They belong to two doublelined eclipsing binaries with resolved orbits: the secondary of the $\mu$ Cas AB system with a mass of $0.17 \mathrm{M}_{\odot}$ (Drummond et al. 1995) and both components of the G 006026 BC system whose masses span 0.43-0.47 $\mathrm{M}_{\odot}$ (Jao et al.2016). 


\section{Physical parameters}

At the time of writing, no mass estimate independent of evolutionary models exist for UCSDs because they are either too faint or no eclipsing/spectroscopic binary exist for direct dynamical mass measurement.

Currently, the range of physical parameters for UCSDs originates from the direct comparison of observed optical and/or near-infrared spectra with state-of-theart evolutionary models. Burgasser et al. (2008) identified a subdwarf with the latest spectral type reported to date (2MASS J0532+8246; sdL7), lying at the stellar/substellar boundary. These authors fitted the full spectral energy distribution (SED) of 2MASS J0532+8246 with the NextGen models (Baraffe et al. 1998), deriving an effective temperature $\left(\mathrm{T}_{\text {eff }}\right)$ of $1730 \pm 90 \mathrm{~K}$ and a mass in the range 0.0744 $0.0835 \mathrm{M}_{\odot}$ for metallicities between 0 and -2.0 dex and ages of 10-15 Gyr. However, the lithium feature at $6707.8 \AA$ has not been detected in higher resolution spectra (Lodieu et al. 2015), suggesting a minimum mass of $0.06 \mathrm{M}_{\odot}$ (Magazzu et al. 1992; Rebolo et al. 1996). Burgasser et al. (2009) repeated a similar process for a warmer L subdwarf classified as sdL4 (Sivarani et al. 2009) and derived a mean $\mathrm{T}_{\text {eff }}$ of $2300 \pm 200 \mathrm{~K}, \log (\mathrm{g})=5.0-5.5 \mathrm{dex}$, and metallicity between -1.0 and $-1.5 \mathrm{dex}$ using the Drift-Phoenix models (Helling et al. 2008). Zhang et al.(2017b) extended such a procedure to six new L subdwarfs identified in the cross-match of SDSS and UKIDSS as well as all previously known L subdwarfs as of 2017. They determined $\mathrm{T}_{\text {eff }}$ and metallicities for $22 \mathrm{~L}$ subdwarfs, extreme subdwarfs, and ultrasubdwarfs with spectral types in the L0-L7 range by direct comparison with the BT-Settl models (Allard et al.2012), yielding temperatures of 1500-2700 K for metallicities between -1.0 and -2.0 dex. On average, the $\mathrm{T}_{\text {eff }}$ of subdwarfs are $100-400 \mathrm{~K}$ higher than solar-metallicity L dwarfs depending on the spectral sub-type and metal class. Some of these L subdwarfs might be brown dwarfs rather than very low-mass stellar members of the halo based on the latest BT-Settl models (Zhang et al. 2017a) but the exact location of the stellar/substellar boundary at low metallicity still requires dynamical measurements.

\section{Future work}

The past two decades have witness the existence of M, L, and T subdwarfs and the first spectral classification of this metal-poor population. The advent of large-scale surveys at both optical and infrared wavelengths (2MASS, SDSS, UKIDSS, WISE) have increased the numbers of UCSDs and extended the sequence to cooler metalpoor L and T dwarfs. However, our knowledge of UCSDs still remains in its infancy. A number of improvements and discoveries are required to take up this field to the next level.

- The improvement in the determination of physical parameters of subdwarfs requires the fitting of optical and infrared spectral energy distributions of several 
hundreds of spectra to complement the extensive work on the properties and kinematics of subdwarfs by Savcheva et al. (2014).

- The saturation of the $\tau_{T i O / C a H}$ index around $\sim \mathrm{M} 8-\mathrm{M} 9$ suggests that a revision of the current spectral classification scheme is needed. Moreover, a near-infrared spectral classification is also necessary to classify future discoveries in largescale infrared surveys.

- The progress in the accuracy of metallicity scale for ultracool subdwarfs calls for searches of close-in and/or wide companions to brighter subdwarfs with welldetermined metallicities.

- Searches for M, L, and T subdwarfs should be enhanced to improve the determination of the object density as a function of metallicity and allow for a determination of the luminosity and mass functions in a distance or magnitude-limited volume.

- The discovery of short-period binaries and eclipsing binaries is heavily needed to infer model-independent masses over a wide range of masses and metallicities.

The future of the field sounds bright with upcoming deep photometric surveys such as the Large Synoptic Survey Telescope (LSST; Ivezic et al.2008) and the Euclid mission (Mellier 2016) and large-scale spectroscopic campaigns planned with WHT/WEAVE (Dalton et al.|2012) and VISTA/4MOST (de Jong et al.2014). Let's look forward to the first substellar subdwarfs and long-waited mass determinations!

\section{References}

Allard F, Homeier D Freytag B (2012) Models of very-low-mass stars, brown dwarfs and exoplanets. Royal Society of London Philosophical Transactions Series A 370:2765-2777

Allers KN Liu MC (2013) A Near-infrared Spectroscopic Study of Young Field Ultracool Dwarfs. ApJ772:79

Allers KN, Jaffe DT, Luhman KL et al. (2007) Characterizing Young Brown Dwarfs Using LowResolution Near-Infrared Spectra. ApJ657:511-520

Baraffe I, Chabrier G, Allard F Hauschildt PH (1998) Evolutionary models for solar metallicity low-mass stars: mass-magnitude relationship and color-magnitude diagrams 337:403-412, URL http://adsabs.harvard.edu/cgi-bin/nph-bib_query? bibcode=1998A\%26A...337 . 403B\&db_key=AST \&high=3d1b390e2520250

Bowler BP, Liu MC Dupuy TJ (2010) SDSS J141624.08+134826.7: A Nearby Blue L Dwarf From the Sloan Digital Sky Survey. ApJ710:45-50

Burgasser AJ (2004) Discovery of a Second L Subdwarf in the Two Micron All Sky Survey. ApJ614:L73-L76

Burgasser AJ Kirkpatrick JD (2006) Discovery of the Coolest Extreme Subdwarf. ApJ645:14851497

Burgasser AJ, Kirkpatrick JD, Brown ME et al. (2002) The Spectra of T Dwarfs. I. Near-Infrared Data and Spectral Classification. ApJ564:421-451

Burgasser AJ, Kirkpatrick JD, Burrows A et al. (2003) The First Substellar Subdwarf? Discovery of a Metal-poor L Dwarf with Halo Kinematics. ApJ592:1186-1192

Burgasser AJ, Geballe TR, Leggett SK, Kirkpatrick JD Golimowski DA (2006) A Unified NearInfrared Spectral Classification Scheme for T Dwarfs. ApJ637:1067-1093

Burgasser AJ, Cruz KL Kirkpatrick JD (2007) Optical Spectroscopy of 2MASS Color-selected Ultracool Subdwarfs. ApJ657:494-510 
Burgasser AJ, Vrba FJ, Lépine S et al. (2008) Parallax and Luminosity Measurements of an L Subdwarf. ApJ672:1159-1166

Burgasser AJ, Witte S, Helling C et al. (2009) Optical and Near-Infrared Spectroscopy of the L Subdwarf SDSS J125637.13-022452.4. ApJ697:148-159

Burningham B, Cardoso CV, Smith L et al. (2013) 76 T dwarfs from the UKIDSS LAS: benchmarks, kinematics and an updated space density. MNRAS433:457-497

Chabrier G (2003) Galactic Stellar and Substellar Initial Mass Function. PASP115:763-795

Cruz KL, Kirkpatrick JD Burgasser AJ (2009) Young L Dwarfs Identified in the Field: A Preliminary Low-Gravity, Optical Spectral Sequence from L0 to L5. AJ137:3345-3357

Cushing MC, Looper D, Burgasser AJ et al. (2009) 2MASS J06164006-6407194: The First Outer Halo L Subdwarf. ApJ696:986-993

Cushing MC, Kirkpatrick JD, Gelino CR et al. (2011) The Discovery of Y Dwarfs using Data from the Wide-field Infrared Survey Explorer (WISE). ApJ743:50

Dalton G, Trager SC, Abrams DC et al. (2012) WEAVE: the next generation wide-field spectroscopy facility for the William Herschel Telescope. In: Ground-based and Airborne Instrumentation for Astronomy IV, Proc SPIE, vol 8446, p 84460P, DOI 10.1117/12.925950

de Jong RS, Barden S, Bellido-Tirado O et al. (2014) 4MOST: 4-metre Multi-Object Spectroscopic Telescope. In: Ground-based and Airborne Instrumentation for Astronomy V, Proc SPIE, vol 9147, p 91470M, DOI 10.1117/12.2055826

Drummond JD, Christou JC Fugate RQ (1995) Full Adaptive Optics Images of ADS 9731 and MU Cassiopeiae: Orbits and Masses. ApJ450:380

Geballe TR, Knapp GR, Leggett SK et al. (2002) Toward Spectral Classification of L and T Dwarfs: Infrared and Optical Spectroscopy and Analysis. ApJ564:466-481

Gizis JE (1997) M-Subdwarfs: Spectroscopic Classification and the Metallicity Scale. AJ113:806822

Gizis JE (1998) High Chromospheric Activity in M Subdwarfs. AJ115:2053-2058

Gizis JE Reid IN (1997) Probing the LHS Catalog. I. New Nearby Stars and the Coolest Subdwarf. PASP109:849-856

Gizis JE, Scholz RD, Irwin M Jahreiss H (1997) APMPM J1523-0245 - A new high proper motion cool subdwarf. MNRAS292:L41-L43

Helling C, Ackerman A, Allard F et al. (2008) A comparison of chemistry and dust cloud formation in ultracool dwarf model atmospheres. MNRAS391:1854-1873

Ivezic Z, Axelrod T, Brandt WN et al. (2008) Large Synoptic Survey Telescope: From Science Drivers To Reference Design. Serbian Astronomical Journal 176:1-13

Jao WC, Henry TJ, Beaulieu TD Subasavage JP (2008) Cool Subdwarf Investigations. I. New Thoughts on the Spectral Types of K and M Subdwarfs. AJ136:840-880

Jao WC, Mason BD, Hartkopf WI, Henry TJ Ramos SN (2009) Cool Subdwarf Investigations. II. Multiplicity. AJ137:3800-3808

Jao WC, Nelan EP, Henry TJ, Franz OG Wasserman LH (2016) Cool Subdwarf Investigations. III. Dynamical Masses of Low-metallicity Subdwarfs. AJ152:153

Joy AH (1947) Radial Velocities and Spectral Types of 181 Dwarf Stars. ApJ105:96

Kirkpatrick JD, Reid IN, Liebert J et al. (1999) Dwarfs Cooler than "M": The Definition of Spectral Type "L" Using Discoveries from the 2 Micron All-Sky Survey (2MASS). ApJ519:802-833, URL http://adsabs.harvard.edu/cgi-bin/ nph-bib_query?bibcode=1999ApJ...519..802K\&db_key=AST

Kirkpatrick JD, Reid IN, Liebert J et al. (2000) 67 Additional L Dwarfs Discovered by the Two Micron All Sky Survey. AJ120:447-472, URL http://adsabs.harvard.edu/ cgi-bin/nph-bib_query?bibcode=2000AJ....120..447K\&db_key=AST

Kirkpatrick JD, Gelino CR, Cushing MC et al. (2012) Further Defining Spectral Type "Y" and Exploring the Low-mass End of the Field Brown Dwarf Mass Function. ApJ753:156

Kirkpatrick JD, Schneider A, FAJardo-Acosta S et al. (2014) The AllWISE Motion Survey and the Quest for Cold Subdwarfs. ApJ783:122

Kirkpatrick JD, Kellogg K, Schneider AC et al. (2016) The AllWISE Motion Survey, Part 2. ApJS224:36 
Leggett SK, Geballe TR, Fan X et al. (2000) The Missing Link: Early Methane ("T”) Dwarfs in the Sloan Digital Sky Survey. ApJ536:L35-L38

Lépine S Scholz RD (2008) Twenty-Three New Ultracool Subdwarfs from the Sloan Digital Sky Survey. ApJ681:L33-L36

Lépine S, Shara MM Rich RM (2003) Discovery of an Ultracool Subdwarf: LSR 1425+7102, the First Star with Spectral Type sdM8.0. ApJ585:L69-L72

Lépine S, Rich RM Shara MM (2007) Revised Metallicity Classes for Low-Mass Stars: Dwarfs (dM), Subdwarfs (sdM), Extreme Subdwarfs (esdM), and Ultrasubdwarfs (usdM). ApJ669:1235-1247

Lodieu N, Scholz RD, McCaughrean MJ et al. (2005) Spectroscopic classification of red high proper motion objects in the Southern Sky. A\&A440:1061-1078

Lodieu N, Zapatero Osorio MR Martín EL (2009) Lucky Imaging of M subdwarfs. A\&A499:729_ 736

Lodieu N, Zapatero Osorio MR, Martín EL, Solano E Aberasturi M (2010) GTC/OSIRIS Spectroscopic Identification of a Faint L Subdwarf in the UKIRT Infrared Deep Sky Survey. ApJ708:L107-L111

Lodieu N, Espinoza Contreras M, Zapatero Osorio MR et al. (2012) New ultracool subdwarfs identified in large-scale surveys using Virtual Observatory tools. I. UKIDSS LAS DR5 vs. SDSS DR7. A\&A542:A105

Lodieu N, Burgasser AJ, Pavlenko Y Rebolo R (2015) A search for lithium in metal-poor L dwarfs. A\&A579:A58

Lodieu N, Espinoza Contreras M, Zapatero Osorio MR et al. (2017) New ultracool subdwarfs identified in large-scale surveys using Virtual Observatory tools. A\&A598:A92

Mace GN, Kirkpatrick JD, Cushing MC et al. (2013) The Exemplar T8 Subdwarf Companion of Wolf 1130. ApJ777:36

Magazzu A, Rebolo R Pavlenko IV (1992) Lithium abundances in classical and weak T Tauri stars. ApJ392:159-171

Martín EL, Delfosse X, Basri G et al. (1999) Spectroscopic Classification of Late-M and L Field Dwarfs. AJ118:2466-2482, URL http://adsabs.harvard.edu/cgi-bin/ nph-bib_query?bibcode=1999AJ....118.2466M\&db_key=AST

Mellier Y (2016) Euclid and the Dark Universe. In: 41st COSPAR Scientific Assembly, abstracts from the meeting that was to be held 30 July - 7 August at the Istanbul Congress Center (ICC), Turkey, but was cancelled. See iA href="http://cospar2016.tubitak.gov.tr/en/" ihttp://cospar2016.tubitak.gov.tr/en/i// $\mathrm{A}_{i}, \quad \mathrm{Ab}$ stract H0.2-1-16., COSPAR Meeting, vol 41

Morgan WW, Keenan PC Kellman E (1943) An atlas of stellar spectra, with an outline of spectral classification. Chicago, Ill., The University of Chicago press

Pinfield DJ, Burningham B, Lodieu N et al. (2012) Discovery of the benchmark metal-poor T8 dwarf BD +01deg2920B. MNRAS422:1922-1932

Rebolo R, Martín EL, Basri G, Marcy GW Zapatero-Osorio MR (1996) Brown Dwarfs in the Pleiades Cluster Confirmed by the Lithium Test. ApJ469:L53

Reid IN Hawley SL (2005) New light on dark stars : red dwarfs, low-mass stars, brown dwarfs. DOI 10.1007/3-540-27610-6

Riaz B, Gizis JE Samaddar D (2008) Hubble Space Telescope Search for M Subdwarf Binaries. ApJ672:1153-1158

Savcheva AS, West AA Bochanski JJ (2014) A New Sample of Cool Subdwarfs from SDSS: Properties and Kinematics. ApJ794:145

Schmidt M (1975) The mass of the galactic halo derived from the luminosity function of highvelocity stars. ApJ 202:22-29

Schmidt SJ, West AA, Burgasser AJ, Bochanski JJ Hawley SL (2010) Discovery of an Unusually Blue L Dwarf Within 10 pc of the Sun. AJ139:1045-1050

Scholz R, Lehmann I, Matute I Zinnecker H (2004a) The nearest cool white dwarf (d 4 pc), the coolest M-type subdwarf (sdM9.5), and other high proper motion discoveries. A\&A425:519527 
Scholz RD, Lodieu N McCaughrean MJ (2004b) SSSPM J1444-2019: An extremely high proper motion, ultracool subdwarf. A\&A428:L25-L28

Schweitzer A, Scholz RD, Stauffer J, Irwin M McCaughrean MJ (1999) APMPM J0559-2903: The coolest extreme subdwarf known. A\&A350:L62-L64

Sivarani T, Lépine S, Kembhavi AK Gupchup J (2009) SDSS J125637-022452: A High Proper Motion L Subdwarf. ApJ694:L140-L143

Zhang ZH, Pinfield DJ, Burningham B et al. (2013) A spectroscopic and proper motion search of Sloan Digital Sky Survey: red subdwarfs in binary systems. MNRAS434:1005-1027

Zhang ZH, Homeier D, Pinfield DJ et al. (2017a) Primeval very low-mass stars and brown dwarfs — II. The most metal-poor substellar object. MNRAS468:261-271

Zhang ZH, Pinfield DJ, Gálvez-Ortiz MC et al. (2017b) Primeval very low-mass stars and brown dwarfs - I. Six new L subdwarfs, classification and atmospheric properties. MNRAS464:3040 3059

Acknowledgements NL is supported by programme AYA2015-69350-C3-2-P from Spanish Ministry of Economy and Competitiveness (MINECO). NL thanks ZengHua Zhang for his input on the review. 